\title{
Original
}

\section{Identification of the Differentially Expressed microRNAs Involved in Cleft Palate Induced by Retinoic Acid (RA) in Mouse Model}

\author{
Chao Wei ${ }^{1}$, Haitao Huang ${ }^{2)}$, Wei Cong ${ }^{1)}$, Zhiguang $\mathrm{Li}^{3)}$, Xuehong Zhang ${ }^{3)}$, Han Liu ${ }^{1)}$, Ru Wang ${ }^{2,4)}$ and Jing Xiao ${ }^{1)}$ \\ ${ }^{1)}$ Department of Oral Pathology, College of Stomatology, Dalian Medical University, Dalian, Liaoning, China \\ 2) Department of Stomatology, the First Affiliated Hospital, Dalian Medical University, Dalian, Liaoning, China \\ ${ }^{3)}$ Institute of Cancer Stem Cell, Cancer Center, Dalian Medical University, Dalian, Liaoning, China \\ 4) Department of Oral and Maxillofacial Surgery, College of Stomatology, Dalian Medical University, Dalian, Liaoning, China \\ (Accepted for publication, April 10, 2018)
}

\begin{abstract}
Identifying the differentially expressed miRNAs in cleft palate, in order to study the molecular mechanism in the development and progress of cleft palate. C57BL/6J mice were used to establish the RA induced cleft palate mouse model. Nine pairs of tissues were obtained on embryonic day 15.5 (E15.5). Total RNA was extracted and miRNAs microarray chip was used to screen the miRNAs. Real-time quantitative PCR (RT-qPCR) was used to verify the miRNAs microarray chip results. Cleft palate related genes targeted by the miRNAs were predicted by TargetScan and miRTarBase, and functional annotation clustering of Gene Ontology (GO) term and KEGG signaling pathways in DAVID were used to analysis these target genes. 1265 miRNAs were identified in cleft palate, and among them 31 were differentially expressed $(p<0.01$, fold change $>1.5)$, including 17 up-regulated and 14 down-regulated miRNAs in cleft palate. 7 up-regulated miRNAs $(m m u-m i R-$ 181a-5p, mmu-miR-410-3p, mmu-miR-3960, mmu-miR-1224-5p, mmu-miR-3970, mmu-let-7e-5p and mmu-miR-1907) and 3 down-regulated miRNAs (mmu-miR-140-3p, mmu-miR-351-5p and mmu-miR-503-5p) were validated by RT-qPCR, and $m m u-$ $m i R-181 a-5 p$ and $m m u-m i R-410-3 p$ were in concordance with those of miRNAs microarray chip detection. 484 target genes were predicted and proven by TargetScan and miRTarBase. GO term showed that RA-induced cleft palate was associated with enrichments in miRNAs involved in embryo development, osteoblast differentiation and so on. KEGG signaling pathways analysis indicated that the differentially expressed miRNAs were involved in MAPK, TGF $\beta$ and WNT signaling pathways. 10 differentially expressed miRNAs in cleft palate have been identified. These miRNAs and their target genes may become new therapeutic targets for cleft palate.
\end{abstract}

Key words: Cleft palate, miRNAs, Retinoic acid, Target genes

\section{Introduction}

Cleft palate, a kind of common human congenital developmental deformities of oral and maxillofacial region, with a prevalence ranging from $1 / 1000$ to $1 / 700$, roots in genetic and environmental disturbances in the development of palate. The formation process of the secondary palatal development, including growth of the palatal shelves, elevation of the palatal shelves, fusion between paired palatal shelves and the disappearance of the midline epithelial seam, is regulated precisely. Disruption at any stage of these steps, involving in growth, elevation or fusion, genetically or environmentally, can cause cleft palate ${ }^{1)}$. This malformation can occur in a non-syndromic form or a syndromic form in conjunction with recognized Mendelian or teratogenic malformations ${ }^{2)}$. Although several genetic and environmental factors have been reported for this disorder, the biological mechanism remains intricate ${ }^{3)}$.

The process of embryonic palatal development in mice is coordinated with the one in mammals temporally and spatially. At embryonic days 12.5 (E12.5) in mice, the palatal shelves root in the maxillary processes as they arise in humans at gestational weeks 6 (GW6), and then grow vertically on both sides of the tongue. At the end of E13.5 (GW8), the palatal shelves elevate to horizon above the tongue. At E14.5 (GW10),

Correspondence to:Dr. Ru Wang, Department of Stomatology, The First Affiliated Hospital, Dalian Medical University, No.222, Zhongshan Road, Dalian, 116011, China; Tel: +86-0411-8363-5963; Fax: +86-0411-8362-2844; E-mail: Wangru@dlmedu.edu.cn.

Dr. Jing Xiao, Department of Oral Pathology, College of Stomatology, Dalian Medical University, No.9, West of Lvshun South Road, Dalian, 116044, China; Tel: +86-0411-8611-0218; Fax: +86-0411-8611-0219; E-mail: xiaoj@dlmedu. edu.cn the medial edge epithelium (MEE) of the palatal shelves begins to contact and adhere between the paired shelves. The adhesive palatal shelves will form a midline epithelial seam (MES). Then fusion is accomplished with mesenchymal confluence by E15.5 (GW12) after the MES rapidly disappears from the midline $e^{4)}$.

Retinoic acid (RA) - a derivative of vitamin A - is the most active compound among retinoids biologically, and it plays important roles in a variety of biological processes, such as cell proliferation and differentiation, regulating morphogenesis of embryonic development, and extracellular matrix production ${ }^{5,6}$. Also, vitamin A and its metabolites (retinoids) are necessary in embryonic development. However, as a medicine for the treatment of cancers and other diseases, RA is a potent teratogen. Excess RA will disrupt the normal development and give rise to the abnormalities of development both in humans and rodents ${ }^{7,8)}$.

miRNAs are a class of highly conserved, approximately 21-25 nucleotides long noncoding RNA molecules that can regulate gene expression at the posttranscriptional level by either degradation or translational repression of a target mRNA ${ }^{9}$. A number of miRNAs have been found in kinds of species and regarded as powerful regulators of gene expression and cellular phenotype. miRNAs are initially transcribed as primary miRNA (pri-miRNA): the pri-miRNA is cleaved in the nucleus by the RNase III enzyme Dicer to produce an 70-nt long precursor miRNA (pre-miRNA) which has a short stem-loop structure ${ }^{10)}$. The pre-miRNA is further cleaved by another RNase III enzyme Dicer to produce the mature miRNA ${ }^{11)}$. The mature miRNA then binds to the RNA-induced silencing complex (RISC), which contains multiple proteins, guiding the RISC interaction with target mRNA sequences, most 


\section{J Hard Tissue Biology Vol. 27(3): 243-249, 2018}

favorably at the 3'untranslated region (3'UTR), suppressing the process of translation and/or causing the degradation of the targeted mRNA $^{9)}$. Growing evidence has suggested that miRNAs participate in a series of biological processes such as cell proliferation, apoptosis, immune response, as well as many diseases ${ }^{12,13)}$.

We used miRNAs microarray chip technology to identify the differentially expressed miRNAs in cleft palate compared with the normal palate tissues in this study, RT-qPCR to verify the accuracy of the miRNAs microarray chip results, TargetScan and miRTarBase to predict and prove the target genes of the differentially expressed miRNAs, and DAVID to analysis the target genes in order to study the molecular mechanism in the development and progress of cleft palate.

\section{Ethics statement}

\section{Materials and Methods}

The Ethics Committee of Dalian Medical University approved all procedures with mice under strict ethical guidelines (L2014034). All experiments with mice abided by the guidelines of the Animal Management Committee of Dalian Medical University. Animals were housed at the Laboratory Animal Center of Dalian Medical University (Animal license SYXK [Liao] 2008-0002). All animals had free access to feed and water at liberty. The facility which accessed by authorized personnel only was controlled for temperature, lighting and ventilation.

\section{Cleft Palate Mouse Model}

Female C57BL/6J mice (10-12-week-old) (Specific pathogen free animal, SPF) were obtained from Laboratory Animal Center of Dalian Medical University and maintained in a specific pathogen free facility at the Laboratory Animal Center of Dalian Medical University. They were used to establish the RA (Sigma Aldrich, St. Louis, USA) induced cleft palate mouse model, in which the pregnant mice (E0 morning vaginal plug was found) were given a single dose of RA at $100 \mathrm{mg} / \mathrm{kg}$ body weight on embryonic day 10.5 (E10.5) respectively. RA was suspended in $0.8 \mathrm{ml}$ corn oil (Yihai kerry food marketing Co. Ltd., Shenzhen, China), and the suspension mixed vigorously to obtain a uniform suspension. The pregnant mice were dosed with $0.2 \mathrm{ml}$ of suspension by gavage, and the control animals were dosed with the same volume of corn oil without RA. Nine pairs of cleft palate and the normal palate tissues were obtained on embryonic day 15.5 (E15.5), saved in $200 \mu$ RNAlater (obtained from LC SCIENCES for free) at $4{ }^{\circ} \mathrm{C}$ overnight and then stored at $-80^{\circ} \mathrm{C}$.

\section{miRNAs microarray chip}

Total RNA was isolated from palate samples of mouse embryo collected on E15.5. The RNA was used to determine the expression of miRNA with a $\mu$ Paraflo ${ }^{\mathrm{TM}}$ mouse MicroRNA microarray, Array 19.0 (LC Sciences, Houston, USA). By adding a poly (A) tail on the 3' end with poly (A) polymerase, total RNA ( $6 \mu \mathrm{g} / \mathrm{sample}$ ) was extended, and then an oligonucleotide tag was ligated to the poly (A) tail for fluorescent dye staining subsequently. The prepared RNA was hybridized to a $\mu$ Paraflo microfluidic chip by incubating overnight under the reaction conditions which were controlled with a micro-circulation pump. With photogenerated reagent chemistry, the detection probes were synthesized in situ. After RNA hybridization, the Cy3 dye bound to the oligo tag for staining through the microfluidic chip. Fluorescence images were acquired with a laser scanner (GenePix 4000B, Molecular Device, California, USA) and digitized with Array-Pro image analysis software (Media Cybernetics, Maryland, USA).

\section{RT-qPCR analysis for miRNAs expression}

Expression levels of mmu-miR-181a-5p, mmu-miR-410-3p, mmu-
miR-3960, mmu-miR-1224-5p, mmu-miR-3970, mmu-let-7e-5p, mmu$m i R-1907, m m u-m i R-140-3 p, m m u-m i R-351-5 p$ and mmu-miR-503$5 p$ were validated using RT-qPCR. The forward and reverse primers were designed by Ribobio. Total RNA was extracted from tissues using RNAlater (obtained from LC SCIENCES for free). A cDNA pool of miRNAs was synthesized by PrimeScript ${ }^{\circledR}$ RT reagent Kit (TaKaRa, Dalian, China) according to the manufacturer's protocol. RT-qPCR was performed using a SYBR ${ }^{\circledR}$ Premix Ex TaqTM II (Perfect Real Time) (TaKaRa, Dalian, China). The reactions were incubated in a $96-$ well optical plate at $95^{\circ} \mathrm{C}$ for $10 \mathrm{~min}$, followed by 45 cycles at $95^{\circ} \mathrm{C}$ for $15 \mathrm{sec}$ and $60^{\circ} \mathrm{C}$ for $1 \mathrm{~min}$. Expression analysis was performed in triplicate for each sample. U6 level was used as internal control.

\section{Target genes prediction, functional annotation clustering of GO term and KEGG signaling pathway analysis}

The prediction of miRNAs target genes is a necessary step in understanding the functions of miRNAs. To further analyze the functions of the differentially expressed miRNAs, we used TargetScan (http:// www.targetscan.org/) ${ }^{14,15)}$ and miRTarBase (http://mirtarbase.mbc. nctu.edu.tw/ $/)^{16,17)}$ to predict their common target genes. To evaluate the target genes for all single miRNAs, we searched for significantly over-represented GO terms $(p<0.01)$ using DAVID Bioinformatics Database (http://david.abcc.ncifcrf.gov/) among all the target genes for the differentially expressed miRNAs respectively ${ }^{18,19)}$. In order to identify the molecular signaling pathways potentially altered by the expression of multiple miRNAs, we used the DAVID Bioinformatics Database. The database performed an enrichment analysis of multiple miRNAs target genes comparing each set of miRNAs targets to all known KEGG (Kyoto Encyclopedia of Genes and Genomes) pathways.

\section{Statistical analysis}

For statistical analysis, data were analyzed using the SPSS 17.0 (IBM, New York, USA). The statistical significance of difference between groups was determined by Student's t-test. Results were considered statistically significant if the $p$-value was less than $0.01(p<0.01)$.

\section{Results \\ miRNAs expression profiles of cleft palate induced by $R A$ in mouse model}

The miRNAs microarray chip differential analysis resulted in 31 differentially expressed miRNAs $(p<0.01$, fold change $>1.5)$ which were significantly expressed in the palate of RA-treated mice compared with controls are shown in Table 1 and Table 2, and the heatmap of differentially expressed miRNAs is plotted and shown in Fig. 1.

\section{Identification of the differentially expressed miRNAs in cleft palate}

In order to verify the results of the miRNAs microarray chip, we chose these differentially expressed miRNAs to be evaluated in matched pairs of cleft palate and the normal palate tissues using RT-qPCR. Of these, 7 up-regulated miRNAs (mmu-miR-181a-5p, mmu-miR-410-3p, mmumiR-3960, mmu-miR-1224-5p, mmu-miR-3970, mmu-let-7e-5p and mmu$m i R-1907)$ and 3 down-regulated miRNAs (mmu-miR-140-3p, mmu$m i R-351-5 p$ and $m m u-m i R-503-5 p$ ) were validated by RT-qPCR, and $m m u-m i R-181 a-5 p$ and $m m u-m i R-410-3 p$ were in concordance with the results of the miRNAs microarray chip data in RA-treated palate tissues compared with controls. RT-qPCR validated the miRNAs microarray chip analysis (Fig. 2).

\section{Target genes prediction of the differentially expressed miRNAs}

In-depth knowledge of the targeted relationship between the 

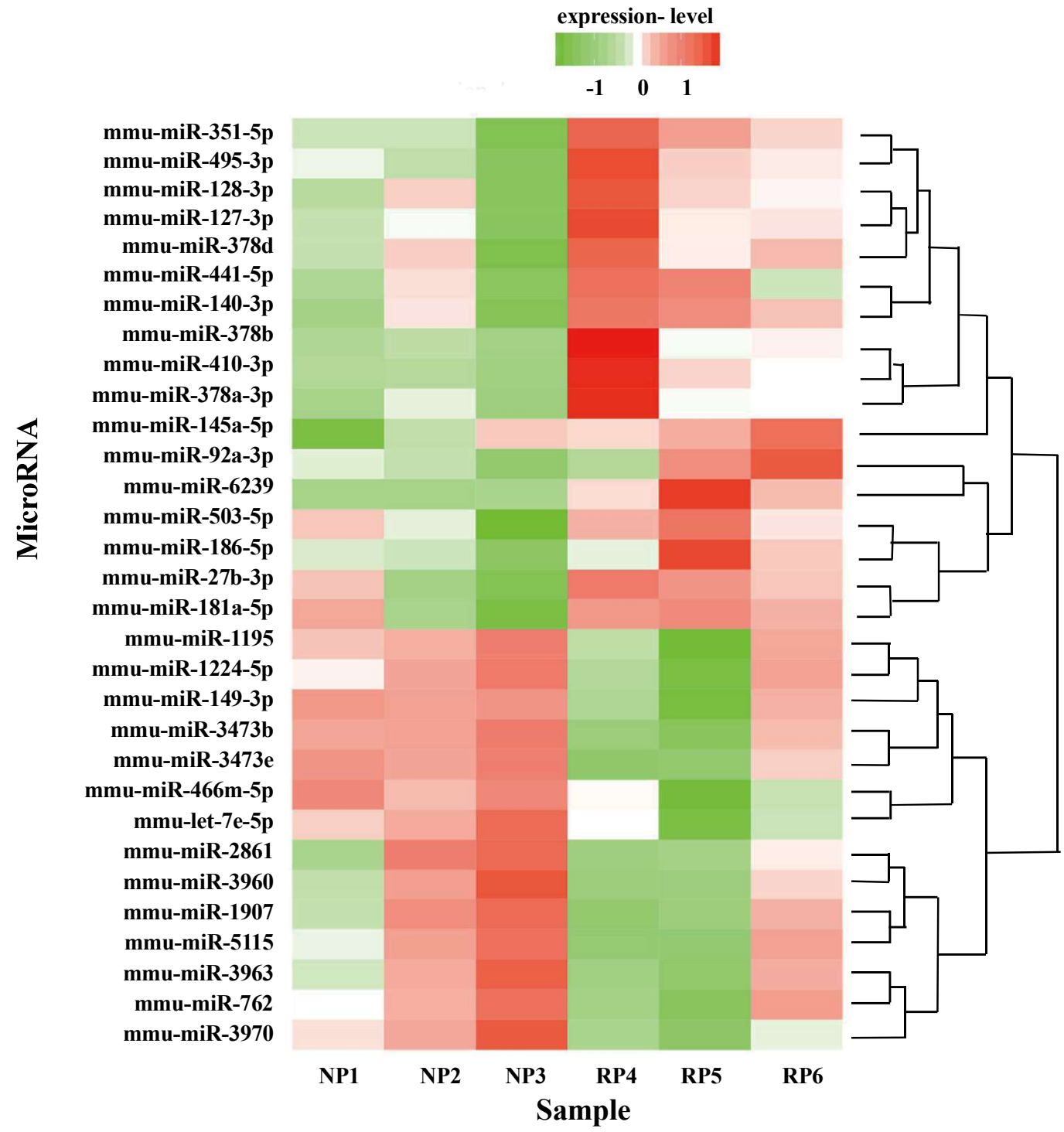

Figure 1. The heatmap of the differentially expressed miRNAs in cleft palate

Differentially expressed miRNAs have been rearranged in this heatmap, where up-regulated and down-regulated miRNAs are clearly displayed $(\mathrm{n}=3 \mathrm{for}$ cleft palate mouse and $n=3$ for control).

Table 1. $p$-value and fold change of 17 up-regulated miRNAs

\begin{tabular}{|c|c|c|}
\hline miRNA & $p$-value & Fold Change \\
\hline$m m u-m i R-140-3 p$ & 7.26E-05 & 2.88 \\
\hline$m m u-m i R-6239$ & $5.55 \mathrm{E}-04$ & 1.66 \\
\hline$m m u-m i R-495-3 p$ & $2.41 \mathrm{E}-03$ & 4.54 \\
\hline$m m u-m i R-181 a-5 p$ & $3.87 \mathrm{E}-03$ & 1.67 \\
\hline$m m u-m i R-127-3 p$ & $3.92 \mathrm{E}-03$ & 3.03 \\
\hline$m т u-m i R-92 a-3 p$ & $8.76 \mathrm{E}-03$ & 1.51 \\
\hline$m m u-m i R-351-5 p$ & $2.40 \mathrm{E}-04$ & 1.84 \\
\hline$m m u-m i R-27 b-3 p$ & $1.00 \mathrm{E}-03$ & 1.83 \\
\hline$m m u-m i R-411-5 p$ & $1.48 \mathrm{E}-03$ & 2.86 \\
\hline$m m u-m i R-145 a-5 p$ & 4.82E-03 & 1.58 \\
\hline$m m u-m i R-410-3 p$ & $5.05 \mathrm{E}-03$ & 2.60 \\
\hline$m m u-m i R-186-5 p$ & $5.15 \mathrm{E}-03$ & 2.08 \\
\hline$m m u-m i R-128-3 p$ & $6.66 \mathrm{E}-03$ & 2.99 \\
\hline$m m u-m i R-503-5 p$ & $7.06 \mathrm{E}-03$ & 1.93 \\
\hline$m m u-m i R-378 d$ & $7.41 \mathrm{E}-03$ & 2.45 \\
\hline$m m u-m i R-378 b$ & $7.71 \mathrm{E}-03$ & 2.52 \\
\hline$m m u-m i R-378 a-3 p$ & $8.02 \mathrm{E}-03$ & 2.47 \\
\hline
\end{tabular}

Table 2. $p$-value and fold change of 14 down-regulated miRNAs

\begin{tabular}{|llc|}
\hline miRNA & $\boldsymbol{p}$-value & Fold Change \\
\hline mmu-miR-3473e & $1.64 \mathrm{E}-04$ & 2.89 \\
$m m u-m i R-3473 b$ & $1.00 \mathrm{E}-03$ & 3.18 \\
mmu-miR-149-3p & $2.23 \mathrm{E}-03$ & 2.97 \\
$m m u-m i R-5115$ & $5.80 \mathrm{E}-03$ & 4.60 \\
mmu-miR-3960 & $5.83 \mathrm{E}-03$ & 6.32 \\
$m m u-m i R-1224-5 p$ & $7.75 \mathrm{E}-03$ & 3.28 \\
mmu-miR-762 & $9.07 \mathrm{E}-03$ & 3.21 \\
mmu-miR-3963 & $9.16 \mathrm{E}-03$ & 6.01 \\
mmu-miR-3970 & $4.44 \mathrm{E}-04$ & 5.05 \\
mmu-let-7e-5p & $1.16 \mathrm{E}-03$ & 2.50 \\
mmu-miR-466m-5p & $4.81 \mathrm{E}-03$ & 2.06 \\
mmu-miR-1907 & $6.11 \mathrm{E}-03$ & 4.47 \\
$m m u-m i R-2861$ & $7.88 \mathrm{E}-03$ & 5.12 \\
mmu-miR-1195 & $8.79 \mathrm{E}-03$ & 2.58 \\
\hline
\end{tabular}




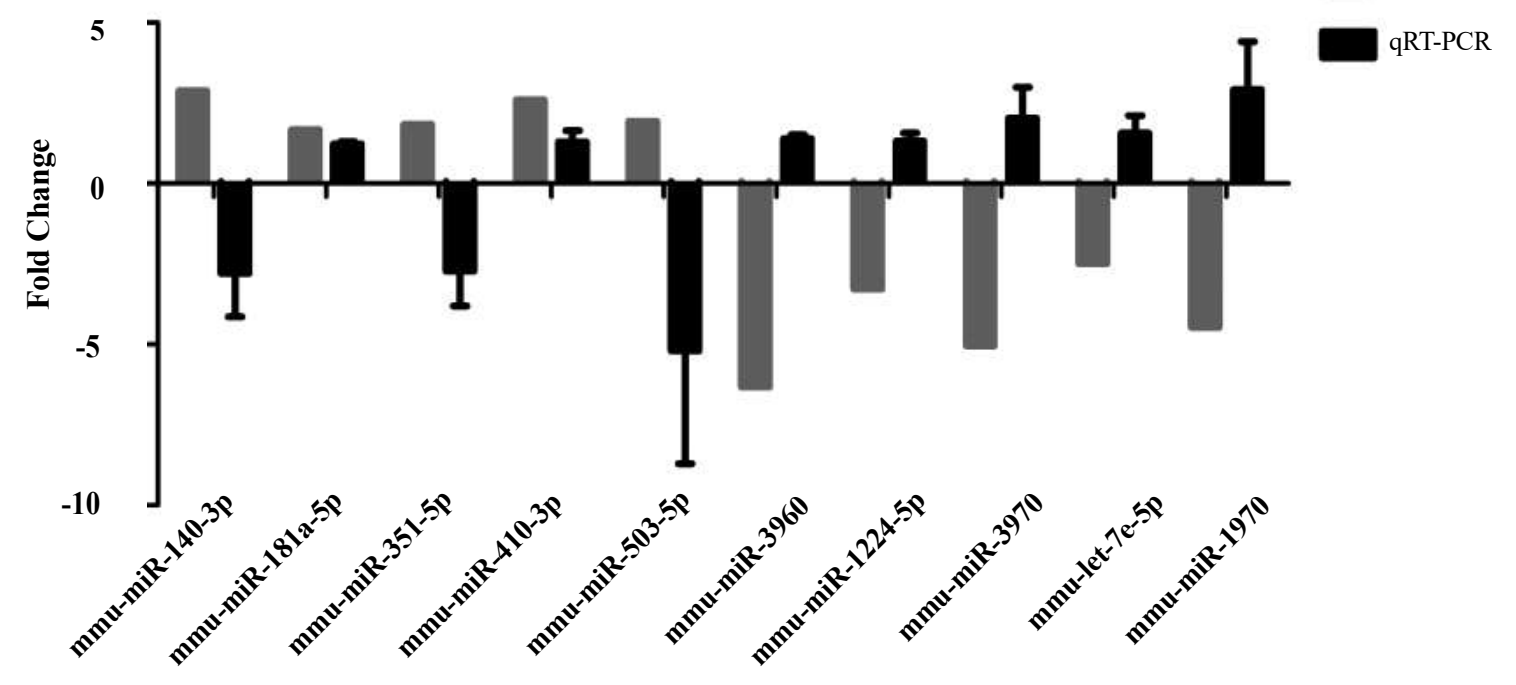

Figure 2. Validation of miRNAs by RT-qPCR

The expression levels of 10 differentially expressed miRNAs were detected by RT-qPCR. The expression levels of 2 differentially expressed miRNAs were in concordance with the normalized miRNAs microarray chip data. Every group was replicated three times using independently collected samples.

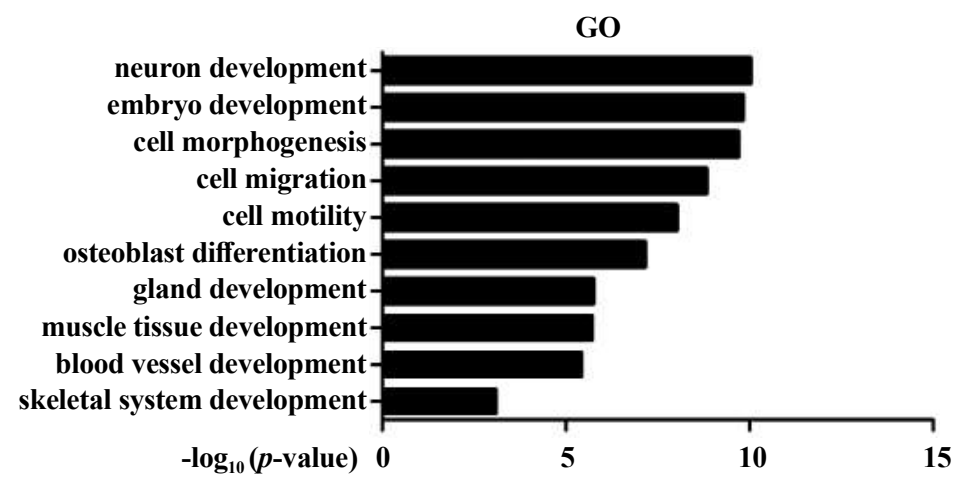

Figure 3. Functional annotation clustering analysis of GO term for target genes of differentially expressed miRNAs

The $\mathrm{x}$-axis indicates the enrichment significance and expressed as the $-\log _{10}$ ( $p$-value), and the $\mathrm{y}$-axis indicates the functional annotation clustering of GO term names.

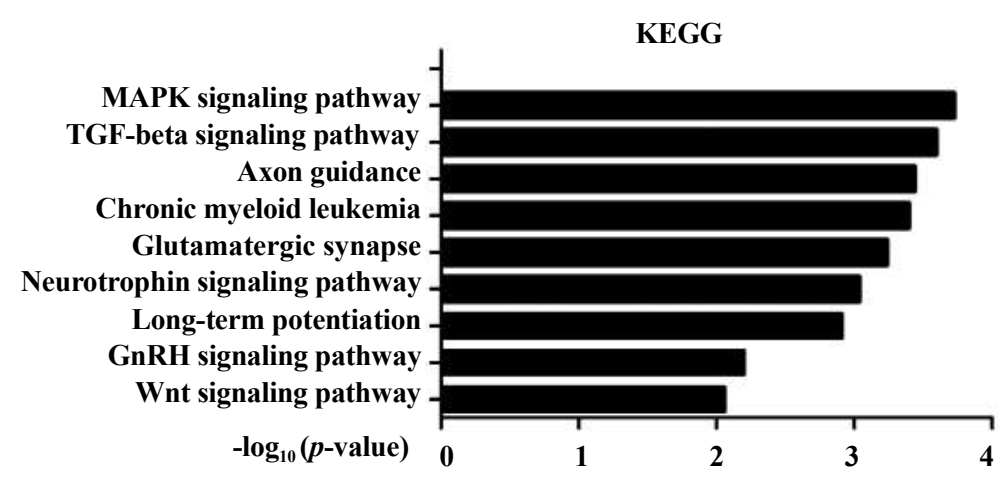

Figure 4. KEGG signaling pathway analysis for target genes of differentially expressed miRNAs

The $\mathrm{x}$-axis indicates the enrichment significance and expressed as the $-\log _{10}$ ( $p$-value), and the $\mathrm{y}$-axis indicates the KEGG signaling pathway names.

differentially expressed miRNAs and genes, TargetScan and miRTarBase was used to predict the target genes of the differentially expressed miRNAs. As expected, these miRNAs could potentially regulate several hundred target genes. Finally, we found out the predicted and proved 484 target genes.

\section{Functional annotation clustering of GO term}

In order to analyze the relationship between expression patterns and functional implications of the target genes, we applied DAVID to classify the target genes of the differentially expressed miRNAs into a couple of function categories. GOTERM_BP_FAT, GOTERM_CC 


\section{Chao Wei et al.: The Differentially Expressed microRNAs in Cleft Plate}

FAT, GOTERM_MF_FAT and SP_PIR_KEYWORDS in Gene Ontology were used to enrich the clusters of related functional terms for all the target genes. We received 19 annotation clusters from the target genes of the differentially expressed miRNAs with the enriched score greater than $3.0(p<0.01)$. Functional annotation clustering analysis of GO term showed that RA-induced cleft palate was associated with enrichments in miRNAs involved in embryo development, cell migration, osteoblast differentiation and so on (Fig. 3).

\section{KEGG signaling pathway analysis}

After analyzing the functional annotation clustering of GO term, we performed the signaling pathway analysis with the target genes of the differentially expressed miRNAs between RA-treated and control palate tissues. KEGG_PATHWAY in Pathways was used to analyze the signaling pathways with the threshold of $p$-value less than 0.01 . The result indicated that the differentially expressed miRNAs between RAinduced cleft palate and control involving in mitogen activated protein kinase (MAPK) signaling, transforming growth factor-beta (TGF $\beta$ ) signaling and WNT/ $\beta$-Catenin signaling pathways were mainly associated with cleft palate (Fig. 4).

\section{Discussion}

Cleft palate and other congenital developmental deformities of oral and maxillofacial region are common in humans and have complex cellular and genetic etiologies. In vertebrates, the palate separates the nasal and oral cavities and is generated through an intricate series of morphogenic events that include early neural crest cell migration and cell-cell signaling during the formation of facial prominences, as well as later generation and fusion of palatal process ${ }^{20}$. Palate development is a complex mechanism that involves elevation, contact, and fusion of palatal process $^{21)}$. Therefore, correct gene expression is necessary for proper palate development in both palatal epithelium and mesenchyme ${ }^{22,23,24)}$.

miRNAs microarray chip, as a kind of rapid and efficient method to analyze the miRNAs expression profiles, has been widely used to identify the differentially expressed miRNAs in tumor ${ }^{25,26)}$. However, there is no report about the miRNAs expression profiles in cleft palate so far. Therefore, we used miRNAs microarray chip technology to identify the differentially expressed miRNAs involved in cleft palate induced by RA in mouse model. Combined with RT-qPCR, we verified 7 up-regulated miRNAs (mmu-miR-181a-5p, mmu-miR-410-3p, mmu-miR-3960, mmumiR-1224-5p, mmu-miR-3970, mmu-let-7e-5p and mmu-miR-1907) and 3 down-regulated miRNAs (mmu-miR-140-3p, mmu-miR-351-5p and mmu$m i R-503-5 p)(p<0.01$, fold change $>1.5)$ in RA-treated palate tissues compared with control.

RA acts as a pivotal role in embryonic development, cell proliferation and differentiation and extracellular matrix production ${ }^{5,6,7)}$. RA could inhibit the growth of MEPM (mouse embryonic palatal mesenchymal) cells in a dose-dependent method by leading to apoptosis. In the cell cycle, RA could bring about a G1 block with a decrease in the proportion of cells in $S$ phase and an increase in the proportion of cells in G0/G1 phase by inhibiting expression of cyclins $\mathrm{D}$ and $\mathrm{E}$ at the protein level ${ }^{27)}$. In mouse fetuses, cleft palate is one of the mainly malformations induced by RA. In RA-induced cleft palate of mouse fetuses, we can see the palatal shelves are still growing vertically on both sides of the tongue. Cell proliferation appeared in epithelium and mesenchyme, and gradually reduced from E13.5 to E15.5. RA didn't influence the proliferation of palatal epithelium. However, cell apoptosis appeared at a specific time and location, and RA could influence the apoptosis of the specific epithelium at E13.5 and E14.5, which was in step with the time of downregulated $\mathrm{Wnt} / \beta$-catenin signaling. As reported, the canonical Wnt/ $\beta$-catenin signaling pathway was completely inhibited by RA at E13.5 and E14.5. Wnt/ $\beta$-catenin signaling pathway was a very important target of RA on account of blocking Wnt/ $\beta$-catenin signaling almost completely

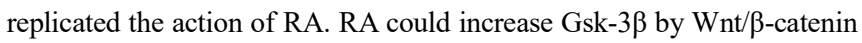
signaling pathway to modulate the development of palate. Furthermore, RAR (RA receptor) is essential for RA to play its biological actions ${ }^{28,29)}$. It has been demonstrated that WNT genes are associated with cleft lip and palate. Wnt3a, Wnt5a and Wnt11 are differentially expressed in non-syndromic cleft lip with or without cleft palate significantly ${ }^{30}$. Autosomal-recessive tetra-amelia with cleft lip and palate is based on mutations in $\mathrm{Wnt}^{31)}$.

TGF $\beta$ (transforming growth factor- $\beta$ ) family members include TGF $\beta 1$, TGF $\beta 2$ and TGF $\beta 3$. In mammals, all the three TGF $\beta$ isoforms are exactly timing and spatial expressed during palatal development. TGF $\beta 3$ seems to act an essential role in all the three TGF $\beta$ isoforms, in which TGF $\beta 3$ gene mutations and/or deficiencies result in cleft palate both in mice ${ }^{32,33,34)}$ and humans ${ }^{35)}$. There are three main types of cell surface receptor proteins in the TGF $\beta 3$ signaling system: TGF $\beta R 1$, TGF $\beta R 2$ and TGF $\beta$ R3. When TGF $\beta$ binds to the cell surface receptor complex, they will lead to phosphorylation and activation of the Smad proteins, which is the intracellular mediators of TGF $\beta$ signaling acting as transcriptional activators of target genes ${ }^{36}$. In the MEPM cells, RA increased the mRNA expression and protein of TGF $\beta 3$, and functional interactions between retinoic systems and TGF $\beta$ had been found in different settings. It had been reported that RA could decrease the expression of TGF $\beta$ R 1 and increase the expression of TGF $\beta R 2$, but couldn't affect the expression of TGF $\beta$ R $3^{28)}$

As reported, $m i R-2861$ accelerated osteoblast differentiation by targeting HDAC5 (histone deacetylase 5), giving rise to increased Runx2 (runt-related transcription factor 2) protein production. miR-3960, clustered with $m i R-2861$, regulated osteoblast differentiation through a regulatory feedback loop with $m i R-2861$. During bone morphogenic protein 2 (BMP2)-induced osteogenesis of ST2 stromal cells, $m i R-3960$ was transcribed. Overexpression of $m i R-3960$ could promote BMP2induced osteoblastogenesis. Furthermore, inhibiting the expression of $m i R-3960$ could attenuate the osteoblastogenesis. Hoxa2 (homeobox A2), which was a repressor of Runx2 expression, was verified to be a target gene of $m i R-3960$. Overexpression of Runx2 could induce $m i R$ 3960/miR-2861 transcription, and inhibiting the expression of Runx2 could attenuate BMP2-induced $m i R-3960 / m i R$ - 2861 transcription ${ }^{37,38)}$. In this study, we speculated that the down-regulated miR-2861 attenuated the osteoblastogenesis of palate bone, which consequently induced cleft palate.

SATB2 (special AT-rich sequence binding protein 2) plays important roles in development and tissue regeneration, especially in palate formation and osteoblast differentiation and so on. During osteoblastogenesis, several cytokines and growth factors regulate the expression of SATB2. Smad1/5 targets SATB2 directly and promotes its expression by BMP2/4/7 activating Smad signaling pathways. BMP2/4/7 also induces Osx and Runx2 expression resulting in SATB2 expression. Osx enhances the expression of SATB2 by binding to the SATB2 promoter directly. In skeletal development and osteoblast differentiation, SATB2 was thought to be an important molecular node. SATB2 can inhibit the expression of Hoxa2 so as to bring about Runx2dependent osteoblast differentiation. SATB2, acts as a transcription factor, can promote key transcription factors and biomarkers of osteoblast differentiation. Both in a Runx2-independent and Runx2-dependent way, SATB2 can induce the expression of Osterix. It can enhance OCN expression by enhancing Runx2 and ATF4 complex, and it also can promote BSP expression by binding to the BSP promoter directly ${ }^{39)}$. 
J Hard Tissue Biology Vol. 27(3): 243-249, 2018

DDIT4 (the DNA-damage-inducible transcript 4), a predicted target gene of miR-181a-5p, suppresses mTOR activity through the TSC1/ TSC2 heterodimer ${ }^{40,41}$. MAPK3 is also another predicted target gene of $m i R-181 a-5 p^{42}$. It is possible that $m i R-181 a-5 p$ inhibits the expression of DDIT4 and MAPK3 to participate in the process of differentiation by mTOR signaling pathway and MAPK/ERK signaling pathway.

In this study, we identified the differentially expressed microRNAs involved in cleft palate induced by RA in mouse model and predicted their target genes. These differentially expressed miRNAs may become new therapeutic targets for cleft palate. On the basis of functional annotation clustering of GO term and KEGG signaling pathway analysis of these differentially expressed microRNAs simultaneously, it helps study the molecular mechanism in the development and progress of cleft palate.

\section{Acknowledgements}

This work was supported by research grants from the National Natural Science Foundation of China (81170944).

\section{Conflict of interest}

The authors have declared that no COI exists.

\section{References}

1. Song Z, Liu C, Iwata J, Gu S, Suzuki A, Sun C, He W, Shu R, Li L, Chai $Y$ and Chen Y. Mice with Tak1 deficiency in neural crest lineage exhibit cleft palate associated with abnormal tongue development. J Biol Chem 288: 10440-10450, 2013

2. Murray JC. Gene/environment causes of cleft lip and/or palate. Clin Genet 61: 248-256, 2002

3. Li L, Zhu GQ, Meng T, Shi JY, Wu J, Xu X and Shi B. Biological and epidemiological evidence of interaction of infant genotypes at Rs7205289 and maternal passive smoking in cleft palate. Am J Med Genet A 155A: 2940-2948, 2011

4. Wu C, Endo M, Yang BH, Radecki MA, Davis PF, Zoltick PW, Spivak RM, Flake AW, Kirschner RE and Nah HD. Intra-amniotic transient transduction of the periderm with a viral vector encoding TGF $\beta 3$ prevents cleft palate in Tgf $33(-/-)$ mouse embryos. Mol Ther 21: 8-17, 2013

5. Wang W and Kirsch T. Retinoic acid stimulates annexin-mediated growth plate chondrocyte mineralization. J Cell Biol 157: 1061-1069, 2002

6. Lai L, Bohnsack BL, Niederreither K and Hirschi KK. Retinoic acid regulates endothelial cell proliferation during vasculogenesis. Development 130: 6465-6474, 2003

7. Ross SA, McCaffery PJ, Drager UC and De Luca LM. Retinoids in embryonal development. Physiol Rev 80: 1021-1054, 2000

8. Mi J. Current treatment strategy of acute promyelocytic leukemia. Front Med 5: 341-347, 2011

9. Bartel DP. MicroRNA: genomics, biogenesis, mechanism, and function. Cell 116: 281-297, 2004

10. Kim VN. MicroRNA precursors in motion: exportin-5 mediates their nuclear export. Trends Cell Biol 14: 156-159, 2004

11. Lee Y, Jeon K, Lee JT, Kim S and Kim VN. MicroRNA maturation: stepwise processing and subcellular localization. Embo J 21: 46634670, 2002

12. Hwang HW and Mendell JT. MicroRNAs in cell proliferation, cell death, and tumorigenesis. Br J Cancer 94: 776-780, 2006

13. Shen H, Liu T, Fu L, Zhao S, Fan B, Cao J and Li X. Identification of microRNAs involved in dexamethasone-induced muscle atrophy. Mol Cell Biochem 381: 105-113, 2013
14. Friedman RC, Farh KK, Burge CB and Bartel DP. Most mammalian mRNAs are conserved targets of microRNAs. Genome Res 19: 92105, 2009

15. Garcia DM, Baek D, Shin C, Bell GW, Grimson A and Bartel DP. Weak seed-pairing stability and high target-site abundance decrease the proficiency of lsy- 6 and other microRNAs. Nat Struct Mol Biol 18: 1139-1146, 2011

16. Hsu SD, Lin FM, Wu WY, Liang C, Huang WC, Chan WL, Tsai WT, Chen GZ, Lee CJ, Chiu CM, Chien CH, Wu MC, Huang CY, Tsou AP and Huang HD. miRTarBase: a database curates experimentally validated microRNA-target interactions. Nucleic Acids Res 39: D163-169, 2011

17. Hsu SD, Tseng YT, Shrestha S, Lin YL, Khaleel A, Chou CH, Chu CF, Huang hy, Lin CM, Ho SY, Jian TY, Lin FM, Chang TH, Weng SL, Liao KW, Liao IE, Liu CC and Huang HD. miRTarBase update 2014: an information resource for experimentally validated miRNAtarget interactions. Nucleic Acids Res 42: D78-85, 2014

18. Xu Y, Zhou B, Wu D, Yin Z and Luo D. Baicalin modulates microRNA expression in UVB irradiated mouse skin. J Biomed Res 26:125-134, 2012

19. Huang da W, Sherman BT and Lempicki RA. Systematic and integrative analysis of large gene lists using DAVID bioinformatics resources. Nat Protoc 4: 44-57, 2009

20. Eberhart JK, He X, Swartz ME, Yan YL, Song H, Boling TC, Kunerth AK, Walker mb, Kimmel CB and Postlethwait JH. MicroRNA Mirn140 modulates Pdgf signaling during palatogenesis. Nat Genet 40: 290-298, 2008

21. Gritli-Linde A. Molecular control of secondary palate development. Dev Biol 301: 309-326, 2007

22. Martínez-Alvarez C, Blanco MJ, Pérez R, Rabadán MA, Aparicio M, Resel E, Martínez T and Nieto MA. Snail family members and cell survival in physiological and pathological cleft palates. Dev Biol 265: 207-218, 2004

23. Cui XM, Chai Y, Chen J, Yamamoto T, Ito Y, Bringas P and Shuler CF. TGF-beta3-dependent SMAD2 phosphorylation and inhibition of MEE proliferation during palatal fusion. Dev Dyn 227: 387-394, 2003

24. Shin JO, Lee JM, Cho KW, Kwak S, Kwon HJ, Lee MJ, Cho SW, Kim KS and Jung HS. MiR-200b is involved in Tgf- $\beta$ signaling to regulate mammalian palate development. Histochem Cell Biol 137:67-78, 2012

25. Avissar M, Christensen BC, Kelsey KT and Marsit CJ. MicroRNA expression ratio is predictive of head and neck squamous cell carcinoma. Clin Cancer Res 15: 2850-2855, 2009

26. Jonigk D, Izykowski N, Maegel L, Schormann E, Maecker-Kolhoff B, Laenger F, Kreipe H and Hussein K. MicroRNA expression in Epstein-Barr virus-associated post-transplant smooth muscle tumours is related to leiomyomatous phenotype. Clin Sarcoma Res 3: 9, 2013

27. Yu Z, Lin J, Xiao Y, Han J, Zhang X, Jia H, Tang Y and Li Y. Induction of cell-cycle arrest by all-trans retinoic acid in mouse embryonic palatal mesenchymal (MEPM) cells. Toxicol Sci 83: 349354, 2005

28. Baroni T, Bellucci C, Lilli C, Pezzetti F, Carinci F, Becchetti E, Carinci P, Stabellini G, Calvitti M, Lumare E and Bodo M. Retinoic acid, GABA-ergic, and TGF-beta signaling systems are involved in human cleft palate fibroblast phenotype. Mol Med 12: 237-245, 2006

29. Hu X, Gao J, Liao Y, Tang S and Lu F. Retinoic acid alters the proliferation and survival of the epithelium and mesenchyme and suppresses Wnt/ $\beta$-catenin signaling in developing cleft palate. Cell Death Dis 4: e898, 2013 
Chao Wei et al.: The Differentially Expressed microRNAs in Cleft Plate

30. Chiquet BT, Blanton SH, Burt A, Ma D, Stal S, Mulliken JB and Hecht JT. Variation in WNT genes is associated with non-syndromic cleft lip with or without cleft palate. Hum Mol Genet 17: 2212-2218, 2008

31. Niemann S, Zhao C, Pascu F, Stahl U, Aulepp U, Niswander L, Weber JL and Müller U. Homozygous WNT3 mutation causes tetraamelia in a large consanguineous family. Am J Hum Genet 74: 558563, 2004

32. Proetzel G, Pawlowski SA, Wiles MV, Yin M, Boivin GP, Howles PN, Ding J, Ferquson MW and Doetschman T. Transforming growth factor-beta 3 is required for secondary palate fusion. Nat Genet 11 : 409-414, 1995

33. Kaartinen V, Voncken JW, Shuler C, Warburton D, Bu D, Heisterkamp N and Groffen J. Abnormal lung development and cleft palate in mice lacking TGF-beta 3 indicates defects of epithelialmesenchymal interaction. Nat Genet 11: 415-421, 1995

34. Brunet CL, Sharpe PM and Ferguson MW. Inhibition of TGF-beta 3 (but not TGF-beta 1 or TGF-beta 2) activity prevents normal mouse embryonic palate fusion. Int J Dev Biol 39: 345-355, 1995

35. Andrew C, Paul A, Ann M, Thomas Doetschman, Nancy J, Sandra Daack-Hirsch, Elena V, Semina, Lisa R, Johnson, Junichiro Machida, Aurora Burds, Timothy J, Parnell, John L.R Rubenstein and Jeffrey C. Murray. Association of MSX1 and TGF 33 with nonsyndromic clefting in humans. Am J Hum Genet 63: 557-568, 1998
36. Massaous J and Hata A. TGF-beta signaling through the Smad pathway. Trends Cell Biol 7: 187-192, 1997

37. Hu R, Liu W, Li H, Yang L, Chen C, Xia ZY, Guo LJ, Xie H, Zhou HD, Wu XP and Luo XH. A Runx2/miR-3960/miR-2861 regulatory feedback loop during mouse osteoblast differentiation. J Biol Chem 286: 12328-12339, 2011

38. Xia ZY, Hu Y, Xie PL, Tang SY, Luo XH, Liao EY, Chen F and Xie H. Runx2/miR-3960/miR-2861 positive feedback loop is responsible for osteogenic transdifferentiation of vascular smooth muscle cells. Biomed Res Int 2015: doi: 10.1155/2015/624037

39. Zhao X, Qu Z, Tickner J, Xu J, Dai K and Zhang X. The role of SATB2 in skeletogenesis and human disease. Cytokine Growth Factor Rev 25: 35-44, 2014

40. Brugarolas J, Lei K, Hurley RL, Manning BD, Reiling JH, Hafen E, Witters LA, Ellisen LW and Kaelin WG Jr. Regulation of mTOR function in response to hypoxia by REDD1 and the TSC1/TSC2 tumor suppressor complex. Genes Dev 18: 2893-2904, 2004

41. DeYoung MP, Horak P, Sofer A, Sgroi D and Ellisen LW. Hypoxia regulates TSC1/2-mTOR signaling and tumor suppression through REDD1-mediated 14-3-3 shuttling. Genes Dev 22: 239-251, 2008

42. Campos C, Sundaram AY, Valente LM, Conceição LE, Engrola S and Fernandes JM. Thermal plasticity of the miRNA transcriptome during Senegalese sole development. BMC Genomics 15: 525, 2014 